\title{
Assessing positive body image, body satisfaction, weight bias, and appearance comparison in emerging adults: A cross-validation study across eight countries
}

\author{
Annie Aimé $^{\mathrm{a}, *}$, Matthew Fuller-Tyszkiewicz ${ }^{\mathrm{b}}$, Jacinthe Dion ${ }^{\mathrm{c}}$, Charlotte Markey ${ }^{\mathrm{d}}$, Esben Strodl ${ }^{\mathrm{e}}$, \\ Marita McCabe $^{\mathrm{f}}$, David Mellor ${ }^{\mathrm{b}}$, Antonio Granero-Gallegose ${ }^{\mathrm{g}}$, Giada Pietrabissa ${ }^{\mathrm{h}, \mathrm{i}}$, \\ Manuel Alcaraz-Ibáneze ${ }^{g}$, Catherine Bégin ${ }^{j}$, Marie-Ève Blackburn ${ }^{k}$, Marie Caltabiano ${ }^{1}$, \\ Gianluca Castelnuovo h,i , Salvatore Gullo ${ }^{\mathrm{m}}$, Naomi Hayami-Chisuwa ${ }^{\mathrm{n}}$, Qiqiang $\mathrm{He}^{\mathrm{o}}$, Gianluca Lo Coco ${ }^{\mathrm{m}}$, \\ Gianmauro Manzonie $^{\mathrm{h}, \mathrm{p}}$, Michel Probst $\mathrm{q}$, Rachel F. Rodgers ${ }^{\mathrm{r}}$, Alvaro Sicilia ${ }^{\mathrm{g}}$, Christophe Maïano ${ }^{\mathrm{a}}$
}

a Department of Psychoeducation and Psychology, Université du Québec en Outaouais, Saint-Jérôme, Québec, Canada

b School of Psychology, Deakin University, 1 Gheringhap Street, Geelong, Victoria, 3220, Australia

${ }^{\mathrm{c}}$ Department of Health Sciences, Université du Québec à Chicoutimi, 555, boul. De l'Université, Chicoutimi, Quebec, G7H 2BI, Canada

d Department of Psychology, Health Sciences Centre, Rutgers University, Camden, NJ, 08102, USA

e School of Psychology and Counselling, Queensland University of Technology, Victoria Park Rd, Kelvin Grove, Queensland, 4059, Australia

f School of Health Sciences, Swinburne University, Burwood Road, Hawthorn, 3122, Australia

${ }^{\mathrm{g}}$ Health Research Center and Department of Education, University of Almeria, Ctra de Sacramento s/n, 04120, Almeria, Spain

${ }^{\mathrm{h}}$ Istituto Auxologico Italiano, IRCCS, San Giuseppe Hospital, Strada Cadorna 90, 28824, Oggebbio, VB, Italy

i Department of Psychology, Catholic University of Milan, Largo Agostino Gemelli, 1, 20123, Milan, Italy

j School of Psychology, Laval University, 2325 Allée des Bibliothèques, Québec, GIV 0A6, Canada

${ }^{\mathrm{k}}$ ECOBES-Research and Transfer, Cégep de Jonquiere, 2505, Saint-Hubert Street, Jonquière, Québec, Canada

${ }^{1}$ School of Psychology, James Cook University, McGregor Road, Smithfield, Queensland, 4870, Australia

${ }^{\mathrm{m}}$ Department of Psychology, Educational Sciences and Human Movement, University of Palermo, viale delle Sciennze edificio 15, 90128, Palermo, Italy

${ }^{n}$ Graduate School of Human Life Sciences, Osaka City University, 3-3-138 Sugimoto Sumiyoshi-ku, Osaka, 558-8585, Japan

o School of Health Sciences, Wuhan University, PR China

p Department of Psychology, eCampus University, Via Isimbardi, 10, 22060, Novedrate, CO, Italy

${ }^{\mathrm{q}}$ Departement of Rehabilitation Sciences, KU Leuven, O\&N4Herestraat 49, 3000, Leuven, Belgium

${ }^{\mathrm{r}}$ Department of Applied Psychology, North Eastern University, 360 Huntington Ave, Boston, MA, 02115, USA

\section{A R T I C L E I N F O}

\section{Article history:}

Received 20 January 2020

Received in revised form 20 September 2020

Accepted 30 September 2020

Available online $\mathrm{xxx}$

Keywords

Positive body image

Emerging adults

Sociodemographic characteristics

\section{A B S T R A C T}

Positive body image refers to individuals' ability to conceptualize their bodies with love, respect, and appreciation. The study of positive body image is relatively new, and instruments used to investigate this multi-faceted construct have received limited use in non-English speaking countries. Thus, the aim of this investigation is to consider four measures that are associated with positive body image across eight different countries. Participants $(n=6272)$ completed the Body Appreciation Scale-2, the Body Areas Satisfaction Scale, the Physical Appearance Comparison Scale, and the Weight Bias Internalization Scale. Multi-group confirmatory factor analyses (MG-CFAs) and item-response theory (IRT) models were used to examine the measurement invariance of these surveys. Our results generally suggest that positive body image, weight bias, and appearance comparison can be

\footnotetext{
* Corresponding author.

E-mail addresses: annie.aime@uqo.ca (A. Aimé); matthew.fuller-tyszkiewicz@deakin. edu.au (M. Fuller-Tyszkiewicz); jacinthe_dion@uqac.ca (J. Dion); chmarkey@camden. rutgers.edu (C. Markey); e.strodl@qut.edu.au (E. Strodl); mmccabe@swin.edu (M. McCabe); david.mellor@deakin.edu.au (D. Mellor); agranero@ual.es (A. Granero-Gallegose); gpietrabissa@gmail.com (G. Pietrabissa); m.alcaraz@ual.es (M. Alcaraz-Ibáneze); catherine.begin@psy.ulaval.ca (C. Bégin); Marie-eveblackburn@ cegepjonquiere.ca (M-È Blackburn); marie.caltabiano@jcu.edu.au (M. Caltabiano); gianluca.castelnuovo@unicatt.it (G. Castelnuovo); salvo.9g@gmail.com (S. Gullo); hayami@life.osaka-cu.ac.jp (N. Hayami-Chisuwa); heqiqiang@gmail.com (Q. He); gianluco.lococo@virgilio.it (G.L. Coco); gianmauro.manzoni@gmail.com (G. Manzonie); Michel.probst@kuleuven.be (M. Probst); R.Rodgers@northeastern.edu (R.F. Rodgers); asicilia@ual.es (A. Sicilia); Christophe.maiano@uqo.ca (C. Maïano)
} 
Cross-validation

Cross-cultural assessed using brief assessments and that these four instruments can be used in different countries, but care should be taken to consider individuals' gender, BMI, and socio-economic position.

\section{Introduction}

Body image is a multi-faceted construct (Andrew, Tiggemann, \& Clark, 2016) encompassing thoughts, beliefs, feelings, and behaviors related to the body (Fiske, Fallon, Blissmer, \& Redding, 2014). Although most available research focuses on the causes and correlates of body dissatisfaction, recent research has included considerations for positive body image (Homan \& Tylka, 2015). Positive body image refers to loving and respectful attitudes towards the unicity and functionality of the body; body acceptance even if one's body does not correspond to the beauty ideals; feelings of confidence, comfort, and happiness with regards to the body; the tendency to put a greater emphasis on the body's assets rather than on its imperfections; and adoption of a broad conceptualization of beauty (Andrew et al., 2016; Halliwell, 2015; Tylka \& Wood-Barcalow, 2015a; Wood-Bacalow, Tylka, \& Augustus-Horvarth, 2010). Positive body image has also been defined as a state of embodiment and a feeling of connection with the body (Halliwell, 2015; Piran, 2015). It implies a capacity and desire to engage in healthy behaviors, attend to one's needs and protect oneself from unrealistic and potentially harmful beauty and appearance-focused messages (Andrew et al., 2016). Although the conceptualization of positive body image has become clearer and has been distinguished from body dissatisfaction over the years, few studies have examined its cross-validation in different countries.

\subsection{Positive body image and reciprocity}

Positive body image encompasses a form of reciprocity (Tylka \& Wood-Barcalow, 2015a) between an individual and the environment. This interdependence means that internal processes (for example, positive body talk or a focus on the functionality of the body) and external sources can influence positive body image (for example, media literacy and support from family or friends; Tylka \& Wood-Barcalow, 2015a). In fact, positive body image is linked to the perception of body acceptance by others (Tylka \& Wood-Barcalow, 2015a). When individuals feel that others find their body acceptable, they are less likely to want to modify it. They may be more prone to accept their bodies and to focus on its functionality. Comments marked by acceptance about the body, infrequent fat- and appearance-talk, and general messages about beauty nurture a positive body image (Tylka \& Wood-Barcalow, 2015a). In contrast, perceptions of non-acceptance by others, conversations about weight loss and different forms of weight stigma, including weight bias, are associated with lower body appreciation (Tylka \& Wood-Barcalow, 2015a; Wasylkiw \& Butler, 2014). Additionally, individuals with low body appreciation may be more likely to over-evaluate beauty ideals, place stronger emphasis on body discrepancies, and compare their appearance with others (Homan \& Tylka, 2015). Thus, for those holding weight bias and using information about others to evaluate their own appearance (i.e., physical appearance comparison), the development and maintenance of positive body image may be hindered.

\subsection{Positive body image and ethnicity}

Past research shows that positive body image differs according to ethnicity. For example, it has been found that different body shapes and sizes tend to be better accepted by African Americans than non-Hispanic Whites (Fallon, Harris, \& Johnson, 2014; Grabe \& Hyde,
2006). In another study examining British female students, Hispanic women reported higher body appreciation than women from other ethnic backgrounds (Swami, Airs, Chouhan, Leon, \& Towell, 2009). More precisely, African Caribbean women came second, Caucasian third and South Asian fourth (Swami et al., 2009). Although positive body image of individuals living in the same country may vary depending on their ethnic backgrounds, possible differences in positive body image across countries have not been studied. Moreover, no study to date has included the assessment of a variety of measures related to positive body image (e.g., body satisfaction, body appreciation, weight bias and physical appearance comparison).

\subsection{Measures of positive body image}

Positive body image was initially operationalized on a continuum of satisfaction/dissatisfaction (Webb, Wood-Barcalow, \& Tylka, 2015), and the Body Areas Satisfaction Subscale (BASS) of the Multidimensional Body-Self Relations Questionnaire (MBSRQ) (Cash, 2000) has long been used to assess it. Given that positive body image is now conceived as a different construct than negative body image or body dissatisfaction and that it is considered an extension of body satisfaction and positive appearance evaluation (Tylka \& Wood-Barcalow, 2015a), other instruments are necessary to more fully capture its essence (Halliwell, 2015) and are essential for theory development and research. Thus, more recently, studies interested in positive body image have used the Body Appreciation Scale (BAS, Avalos, Tylka, \& Wood-Barcalow, 2005; BAS-2, Tylka \& Wood-Barcalow, 2015b). The psychometric properties of the BAS-2, a revised version of the BAS, were first tested among U.S. samples of college and community women and men (Tylka \& Wood-Barcalow, 2015b). In these samples, adequate internal consistency coefficients and good stability over a three-week period were found. A one-dimensional factor structure was found in non-English speaking adults from Hong Kong, Iran, the Netherlands, Serbia, China, France, Japan, Romania, Malaysia and the United Arab Emirates (see Swami et al., 2019). However, data regarding the measurement invariance of the BAS-2 between various geographic regions has not been examined (Swami et al., 2019; Webb et al., 2015). Yet, body appreciation may not be conceived and expressed similarly across countries.

\subsection{Current study}

Past research has focused on positive body image mostly used English- speaking and college samples, female sub-populations and/or samples from the U.S. (Halliwell, 2015). The factor structure of the BASS of the MBSRQ was confirmed in a French sample (Untas, Koleck, Rascle, \& Borteyrou, 2009), but this scale was not invariant across black and white women (Kelly et al., 2012). These results suggest that more research is needed to test the invariance of this measure and to determine if it can be used for cross-country comparisons (Fiske et al., 2014). Further, most conclusions concerning positive body image relying on the BAS are equivocal with regards to its factor structure, with some studies suggesting a unidimensional factor structure and others a bidimensional one (Halliwell, 2015). The cross-cultural unidimensional structure of the revised version of the BAS, the BAS-2, still needs to be evaluated. Moreover, measures other than the BAS-2 should also be used to better understand positive body image across different countries. Given the multidimensional nature of the positive body image concept as well as the reciprocity between individual and environmen- 
tal factors in forming this concept, it appears important to determine the cross-country measurement invariance of different questionnaires used to assess positive body image. These questionnaires not only assess more typical components of positive body image, such as body satisfaction and appreciation, but also components known to play a role in its development and maintenance, such as physical appearance comparison and weight bias.

Thus, additional cross-validation efforts are necessary because: (1) the validity and factorial structure of the questionnaires used to measure positive body image may vary across samples composed of women and men as well as of people living in non-English speaking countries; and (2) the question as to whether these questionnaires can be used for comparison among samples from different countries remains unanswered.

The aim of this research is therefore to validate two measures of positive body image and two measures of constructs related to positive body image (e.g. physical appearance comparison and weight bias), and evaluate their measurement invariance across different countries and languages. More specifically, this cross-validation study examines the factorial validity and reliability of the BAS-2, BASS, the Physical Appearance Comparison Scale (PACS; Thompson, Heinberg, \& Tantleff-Dunn, 1991), and the Weight Bias Internalization Scale (WBIS-M; Pearl \& Puhl, 2014) in a sample composed of emerging adults aged between 18-30 years from eight different countries, representing seven different languages. Emerging adulthood represents a developmental period that follows adolescence and precedes adulthood. During this developmental period, youth experience various changes, including possible weight gain and body changes putting them at risk of body dissatisfaction (Arnett, Žukauskienė, \& Sugimura, 2014). Although developmental research on positive body image is limited (See Rodgers et al., 2017), it seems possible that emerging adults are at particular risk of failing to experience their bodies in a positive manner.

\section{Method}

\subsection{Participants}

This sample was comprised of 6272 participants (4218 females, 1928 males, and 24 missing data for gender) aged 18-30 years old, living in eight different countries: Australia $(n=596)$, Belgium $(n=618)$, Canada $(n=766)$, China $(n=663)$, Italy $(n=660)$, Japan $(n=622)$, Spain $(n=821)$, and the United States $(n=1515)$. They were mostly single $(60.9 \%)$ or involved in a relationship (34.3\%). Their mean age was $21.55 \pm 3.13$ and average BMI of $23.30 \pm 4.94 \mathrm{~kg} / \mathrm{m}^{2}$.

\subsection{Procedure}

Since the measures used in this study were all developed in English, and this is not the first language of participants residing in Belgium, Canada (French speaking participants), China, Italy, Japan, and Spain, several versions of the measures were used. When the measures were not available in one of the non-English languages (i.e., either in Chinese, Dutch, French, Italian, Japanese, or Spanish), an iterative process of independent forward- and back-translation was undertaken. A detailed description of the translation process and procedure can be found elsewhere (see McCabe et al., 2019).

Ethics approval for the project and data management were obtained (approval number: 2017-259E). In addition, researchers from each of the eight countries obtained ethics approval from their university ethics committees. Recruitment was conducted through social media sites, online forums, and mailing lists. Participation occurred only online, through the survey software Qualtrics. Participants provided digital informed consent. In some settings, participants were incentivized by the chance to win a modest financial prize or with class credit. The survey was anonymous and lasted approximately 60-90 min. Data collection took part between July 2018 and March 2019 (for more information, see McCabe et al., 2019).

\subsection{Measures}

\subsubsection{Demographic predictors}

Participants from all countries were asked to self-report their age, gender (coded 0 for men and 1 for women), height and weight, and perceived socioeconomic status (SES). Self-reported height and weight were used to calculate body mass index (BMI). The Subjective Social Status scale was used to assess perceived SES using a scale ranging from 1 to 10; participants had to represent themselves on this scale where 1 represents people who have the least money, least education, and the least respected jobs or no job; 10 represents those who have the most money, the most education, and the most respected jobs (Operario, Adler, \& Williams, 2004).

\subsubsection{Body appreciation}

Body appreciation was evaluated with the Body Appreciation Scale-2 (BAS-2; Tylka \& Wood-Barcalow, 2015a, 2015b). This 10-item measure asks participants to indicate, on a 5-point scale, how true the statements are for them. Example items include: 'I respect my body' and 'I am comfortable with my body'. The BAS- 2 items were averaged to compute a total score, ranging from 1 (never) to 5 (always). Higher scores indicate greater body appreciation.

\subsubsection{Body satisfaction}

The Body Areas Satisfaction Scale (BASS; Brown, Cash, \& Mikulka, 1990; Cash, 2000) of the Multidimensional Body-Self Relations Questionnaire (MBSRQ) was used to assess body satisfaction. It is composed of nine items assessing the degree of satisfaction/dissatisfaction with eight different aspects of one's appearance (i.e., face, hair, lower torso, mi torso, upper torso, muscle tone, weight, and height) as well as overall appearance, using a 5-point Likert-type scale, ranging from 1 (very dissatisfied) to 5 (very satisfied). In this sample, the nine BASS items were averaged to compute a total score.

A higher score points to contentment with most areas of the body, a lower score indicates dissatisfaction with the size and appearance of several areas. In this sample, the nine BASS items were averaged to compute a total score.

\subsubsection{Physical appearance comparison}

The Physical Appearance Comparison Scale (PACS; Thompson et al., 1991) is a 5-item questionnaire measuring general appearance comparison frequency on a 4-point scale, ranging from 1 (never) to 4 (always). These items include: "At parties or other social events, I compare my physical appearance to the physical appearance of others" and "In social situations, I sometimes compare my figure to the figures of other people." A total score is calculated by summing the scores of each item. A higher total score indicates higher frequency of appearance comparison.

\subsubsection{Weight bias}

Internalization of weight bias was evaluated with a modified version of the Weight Bias Internalization Scale (WBIS-M; Pearl \& Puhl, 2014). This 11-item measure was adapted from the original version (Durso \& Latner, 2008) in order to make it accessible to respondents of various weight categories. Example items are: "I hate myself for my weight" and "Because of my weight, I don't understand how anyone attractive would want to date me." As with the original scale, participants answered on a 7-point scale, ranging from 1 (strongly disagree) to 7 (strongly agree). The 11 items of the modified WBIS-M were averaged to produce a total score. 


\subsection{Analyses}

All analyses were performed using Mplus 8.3 (Muthén \& Muthén, 2018) and the robust maximum likelihood (MLR) estimator. Additionally, full information maximum likelihood was used to account for the limited level of missing data present at the item level (BAS-2: 0.02 $\%-0.22 \%$ of missing data per item, $M=0.13 \%, S D=.06 \%$; BASS: $0.02 \%-0.17 \%$ of missing data per item, $M=0.11 \%, S D=0.05 \%$; PACS: $0.14 \%-0.31 \%$ of missing data per item, $M=0.18 \%, S D=0.12$ $\%$; WBIS-M: $0.17 \%-0.50 \%$ of missing data per item, $M=0.33 \%$, $S D=0.11 \%)$.

In the first stage, an a priori unidimensional factor structure of the BAS-2, BASS, PACS, and WBIS-M was examined with a confirmatory factor analytic (CFA) model. For each questionnaire, the a priori one-factor CFA models comprise the total number of items in which error terms would be uncorrelated. The composite reliability of the factor of the BAS-2, BASS, PACS, and WBIS-M were estimated using McDonald'S (1970) omega $(\omega)$.

Model fit was based on the following goodness of fit indices (e.g., Marsh, Hau, \& Grayson, 2005; Schermelleh-Engel, Moosbrugger, \& Müller, 2003): the comparative fit index (CFI) and Tucker-Lewis index (TLI; TLI/CFI $\geq .97$ indicates good fit; $\geq .95$ indicates acceptable fit), the root mean square error of approximation (RMSEA; RMSEA $\leq .05$ indicates good fit; $\leq .08$ indicates acceptable fit), and the $90 \%$ confidence interval of the RMSEA. The MLR chi-square test was also presented. However, because this indicator is oversensitive to sample size and minor misspecifications (e.g., Hu \& Bentler, 1999; Marsh et al., 2005), we did not rely on this indicator for model estimation. In general, several strategies were used to improve the factor structure in the event of poor fit for the initially proposed models: (1) deletion of items either with communality values $<.3$ or showing high collinearity with other items (i.e., $r>$.80), and (2) addition of covariances among residual variances of items based on modification indices and theoretical reasonableness of their inclusion.

In the second stage, the measurement invariance of the most appropriate factor structure (retained in the first step) of the BAS-2, BASS, PACS, and WBIS-M were examined, across countries (i.e., Australia, Belgium, Canada, China, Italy, Japan, Spain and United States of America), consistent with procedures recommended by Meredith (1993): (a) configural (item/factor clusters) invariance, i.e., each common factor is associated with the same items across countries (loadings, intercepts, and uniquenesses (residual variances) are freely estimated, latent variances are constrained to 1 , and latent means are constrained to 0 in all groups); (b) loadings (metric) invariance, i.e., corresponding factor loadings are equal across countries (also named weak invariance; the loadings are constrained to invariance, the variances are freely estimated in all but one group, and the latent means are constrained to 0 in all groups); (c) intercept invariance, i.e., considering differential additive response bias (or acquiescent response styles) (also named strong invariance; the intercepts are constrained to invariance, the variances are freely estimated in all but one group, and the latent means are freely estimated in all but one group); (d) uniquenesses invariance (also named strict invariance); (e) correlated uniqueness (if applicable) invariance; (f) invariance of latent variance; and (g) latent mean invariance. In each sequence of invariance, the preceding model served as comparison. As recommended in the literature (e.g., Chen, 2007; Cheung \& Rensvold, 2002), all model comparisons were based on changes $(\Delta)$ in CFIs, TLIs an RMSEAs. Therefore, there is measurement invariance when $\Delta$ CFIs/ TLIs $\leq .01$ and $\triangle$ RMSEAs $\leq .015$.

In a third stage, as recommended by Morin et al. (2018), a hybrid multiple indicators multiple causes (MIMIC) multiple-group model was used to examine: (a) the associations between demographic predictors (i.e., age, BMI, sex, and SES) and scores on the latent factor of the
BASS, BAS-2, WBIS-M, and PACS; (b) possible differential item functioning (DIF) as a function of these demographic predictors (i.e., direct effects between the predictors and item response over and above the effects of the predictors on the latent factor); and (c) the invariance of these associations across countries. As recommended by Morin et al. (2018), for each questionnaire (i.e., BAS-2, BASS, PACS, and WBIS-M), these models were built from the most invariant multiple-group model identified in the second stage, to which the demographic predictors were added.

More precisely, hybrid MIMIC models were examined in the following sequence (Marsh, Nagengast, \& Morin, 2013; Morin, Marsh, \& Nagengast, 2013): (a) null effects model (the paths from the demographic predictors to the latent factor and to the item responses are constrained to be zero); (b) saturated model (the paths from the demographic predictors to the item responses are freely estimated, while the paths from the demographic predictors to the latent factor are constrained to be zero); and (c) factors only model (the paths from the demographic predictors to the latent factor are freely estimated, while the paths from the demographic predictors to the item responses are constrained to be zero). To facilitate interpretation, age, BMI, and SES were standardized prior to the analyses.

As recommended by Morin et al. (2018), a substantial improvement in model fit (i.e., $\Delta$ CFIs/TLIs $\geq .01$ and $\triangle$ RMSEAs $\geq .015$ ) associated with the factor only and saturated models in comparison to the null effects models support the presence of relations between the demographic predictors and item responses. However, improvement in model fit associated with the saturated model when compared to the factors only model provides support for the presence of DIF. These models were first examined while allowing all associations to be freely estimated (or equally constrained to be zero) across countries. Then, the most appropriate model was retained and compared to an alternative model in which all associations were constrained to be equal (i.e., invariant) across countries.

\section{Results}

\subsection{Factor validity and reliability of the BASS, BAS-2, WBIS-M, and PACS}

The goodness-of-fit statistics of CFA for the BASS, BAS-2, WBIS-M, and PACS are displayed in Table 1 (see models $1-1$ to $1-7$ ).

\subsubsection{BAS-2}

As presented in Table 1 (see model 1-1), the a priori one-factor CFA resulted in an acceptable (for RMSEA) to good (for CFI/TLI) level of fit. The standardized parameter estimates from this model, which are presented in Table 2, show that all loadings are substantial $\left(M_{\lambda}=.815\right.$; ranging from .717 to .896). Finally, as presented in Table 2, the BAS-2 showed an excellent composite reliability.

\subsubsection{BASS}

The a priori one-factor CFA resulted in a poor (for all indices) level of fit (see model 1-2). In order to improve the a priori model, we correlated the residual variances of Items 2 ["Hair (colour, thickness, texture)"] and 1 ["Face (facial features, complexion)"], 7 ["Weight"] and 4 ["Mid torso (waist, stomach)"], and 9 ["Overall appearance"] and 1 ["Face (facial features, complexion)"]. This alternative model (see models $1-3$ ) including three correlated residual variances resulted in a substantial improvement of model fit indices and provided an acceptable (for RMSEA and TLI) to good (for CFI) level of fit to the data. The standardized parameter estimates from this model, presented in Table 2, revealed that all loadings are acceptable $\left(M_{\lambda}=.617\right.$; ranging from .363 to .821$)$. The correlation between the residual variances were significant (at $p<.001)$, positive and modest $(M=.310$; ranging from .269 
Table 1

Goodness-of-Fit Statistics of Confirmatory Factor Analyses (CFA) for the BAS-2, BASS, PACS and WBIS-M

\begin{tabular}{|c|c|c|c|c|c|c|c|c|c|c|c|c|c|c|c|c|}
\hline \multirow{2}{*}{$\begin{array}{l}\text { Questionnaires } \\
\text { BAS-2 }\end{array}$} & \multirow{2}{*}{$\begin{array}{l}\text { Models } \\
\text { CFA }\end{array}$} & \multirow{2}{*}{$\frac{\mathrm{N}^{\circ}}{1-1}$} & \multirow{2}{*}{$\begin{array}{l}\text { Description } \\
\text { Overall sample }(n=5955)\end{array}$} & \multicolumn{2}{|l|}{$\chi^{2}(\mathrm{df})$} & \multirow{2}{*}{$\begin{array}{l}\text { CFI } \\
.967\end{array}$} & \multirow{2}{*}{$\begin{array}{l}\text { TLI } \\
.958\end{array}$} & \multirow{2}{*}{$\begin{array}{l}\text { RMSEA } \\
.072\end{array}$} & \multicolumn{2}{|c|}{ RMSEA 90 \% CI } & \multirow{2}{*}{ CM } & \multicolumn{2}{|c|}{$\Delta \mathrm{R} \chi^{2}(\mathrm{df})$} & $\Delta \mathrm{CFI}$ & $\Delta$ TLI & $\triangle$ RMSEA \\
\hline & & & & \multirow{2}{*}{\multicolumn{2}{|c|}{$\begin{array}{l}1123.641(35)^{* * *} \\
2170.838(27)^{* * *}\end{array}$}} & & & & \multicolumn{2}{|c|}{$.069-.076$} & & \multicolumn{2}{|l|}{ - } & - & - & - \\
\hline \multirow[t]{2}{*}{ BASS } & CFA & $1-2$ & Overall sample $(n=6046)$ & & & .873 & .831 & .115 & .111 & -.119 & - & - & & - & - & - \\
\hline & $\mathrm{CFA}+\mathrm{CU}$ & $1-3$ & Overall sample $(n=6046)$ & 767.14 & $24) * * *$ & .956 & .934 & .072 & .067 & -.076 & $1-2$ & - & & +.083 & .103 & -.043 \\
\hline \multirow[t]{2}{*}{ PACS } & CFA - 5 items & $1-4$ & Overall sample $(n=5763)$ & 171.80 & $5)^{* * * *}$ & .979 & .959 & .076 & .067 & -.086 & - & - & & - & - & - \\
\hline & CFA - 4 items & $1-5$ & Overall sample $(n=5763)$ & $1.135(2$ & & 1.00 & 1.00 & .000 & .000 & -.002 & $1-4$ & - & & +.021 & +.041 & -.076 \\
\hline WBIS-M & CFA - 11 items & $1-6$ & Overall sample $(n=5790)$ & 2697.6 & $(44)^{* * * *}$ & .909 & .887 & .102 & .099 & -.105 & - & - & & - & - & - \\
\hline & CFA -10 items + CU & $1-7$ & Overall sample $(n=5786)$ & 1053.7 & $3(30) * * *$ & .962 & .942 & .077 & .073 & -.081 & $1-6$ & - & & +.055 & +.058 & -.027 \\
\hline BAS-2 & MI & $2-1$ & Configural invariance & 1719.7 & $3(280)^{* * * *}$ & .957 & .945 & .083 & .079 & -.087 & - & - & & - & - & - \\
\hline & & $2-2$ & Weak invariance & 2135.8 & $2(343) * * * *$ & .947 & .944 & .084 & .080 & -.087 & $2-1$ & $426 . \varepsilon$ & $(63)^{* * * * *}$ & -.010 & -.001 & .001 \\
\hline & & $2-3$ & Strong invariance & 3535.7 & $(406)^{* * * *}$ & .907 & .917 & .102 & .099 & -.105 & $2-2$ & 1603 & $7(63)^{* * * *}$ & -.040 & -.027 & +.018 \\
\hline & & $2-4$ & Partial strong invariance & 2357.1 & $(391)^{* * * *}$ & .941 & .946 & .082 & .079 & -.085 & $2-2$ & 203. & $(48)^{* * * * *}$ & -.006 & +.002 & -.002 \\
\hline & & $2-5$ & Strict invariance & 3059.5 & $(461)^{* * * *}$ & .923 & .940 & .087 & .084 & -.090 & $2-4$ & 655.1 & $(70)^{* * * *}$ & -.018 & -.006 & +.005 \\
\hline & & $2-6$ & Partial strict invariance & 2633.9 & $(454)^{* * * * *}$ & .935 & .948 & .080 & .077 & -.083 & $2-4$ & 296.2 & $(63) * * * *$ & -.006 & +.002 & -.002 \\
\hline & & $2-7$ & Variance invariance & 2716.4 & $2(461)^{* * * *}$ & .933 & .948 & .081 & .078 & -.084 & $2-6$ & 98.55 & 7)**** & -.002 & .000 & +.001 \\
\hline & & $2-8$ & Latent mean invariance & $3246.1 \varepsilon$ & $(468)^{* * * * *}$ & .917 & .936 & .089 & .086 & -.092 & $2-7$ & 656. & $(7) * * * *$ & -.016 & -.012 & +.008 \\
\hline BASS & MI & $3-1$ & Configural invariance & 917.52 & 192)*** & .955 & .932 & .071 & .066 & -.075 & - & - & & - & - & - \\
\hline & & $3-2$ & Weak invariance & 1114.6 & $(248)^{* * * *}$ & .946 & .937 & .068 & .064 & -.072 & $3-1$ & $185 . \varepsilon$ & 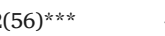 & -.009 & +.005 & -.003 \\
\hline & & $3-3$ & Strong invariance & 2243.7 & $(304)^{* * * *}$ & .878 & .885 & .092 & .088 & -.095 & $3-2$ & 1220 & $37(56) * * *$ & -.068 & -.052 & +.024 \\
\hline & & $3-4$ & Partial strong invariance & $1274.9 ?$ & $3(285) * * * *$ & .938 & .937 & .068 & .064 & -.072 & $3-2$ & 159.6 & $(37) * * * *$ & -.008 & .000 & .000 \\
\hline & & $3-5$ & Strict invariance & 1671.1 & $(348)^{* * * *}$ & .917 & .931 & .071 & .068 & -.074 & $3-4$ & $406 . c$ & $(63)^{* * * *}$ & -.021 & -.006 & +.003 \\
\hline & & $3-6$ & Partial strict invariance & 1443.7 & $(332) * * *$ & .930 & .940 & .067 & .063 & -.070 & $3-4$ & 165.6 & $3(47) * * * *$ & -.008 & +.003 & -.001 \\
\hline & & $3-7$ & $\mathrm{CU}$ invariance & 1480.7 & $(353) * * *$ & .929 & .942 & .065 & .062 & -.068 & $3-6$ & 48.80 & $21)^{* * * * *}$ & -.001 & +.002 & -.002 \\
\hline & & $3-8$ & Variance invariance & 1549.6 & $3(360) * * *$ & .925 & .940 & .066 & .063 & -.070 & $3-7$ & 76.14 & 7)**** & -.004 & -.002 & +.001 \\
\hline & & $3-9$ & Latent mean invariance & 2143.3 & $(367)^{* * * *}$ & .889 & .913 & .080 & $.07 \%$ & -.083 & $3-8$ & 679.1 & $(7) * * *$ & -.036 & -.027 & +.014 \\
\hline PACS & MI & $4-1$ & Configural invariance & 46.866 & 6)***k & .995 & .986 & .052 & & -.069 & - & - & & - & - & - \\
\hline & & $4-2$ & Weak invariance & 302.72 & $37)^{* * *}$ & .959 & .947 & .100 & .09 & -.110 & $4-1$ & 260.1 & $(21)^{* * * *}$ & -.036 & -.039 & +.048 \\
\hline & & $4-3$ & Partial weak invariance & 100.75 & $33)^{* * *}$ & .990 & .985 & .053 & & -.065 & $4-1$ & 54.11 & $17)^{* * * *}$ & -.005 & -.001 & +.001 \\
\hline & & $4-4$ & Strong invariance & 1085.1 & $(54)^{* * * *}$ & .841 & .858 & .163 & .15 & -.171 & $4-3$ & 974.8 & $(21)^{* * * *}$ & -.149 & -.127 & +.110 \\
\hline & & $4-5$ & Partial strong invariance & 143.44 & $47)^{* * *}$ & .985 & .985 & .053 & & -.063 & $4-3$ & 42.65 & $14)^{* * *}$ & -.005 & .000 & .000 \\
\hline & & $4-6$ & Strict invariance & 373.86 & $75)^{* * *}$ & .954 & .970 & .074 & & -.082 & $4-5$ & 193. & $(28)^{* * * *}$ & -.031 & -.015 & +.021 \\
\hline & & $4-7$ & Partial strict invariance & 183.72 & $65)^{* * * *}$ & .982 & .986 & .050 & & -.059 & $4-5$ & 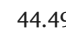 & $18)^{* * * *}$ & -.003 & +.001 & -.003 \\
\hline & & $4-8$ & Variance invariance & 230.48 & $72 * * * *$ & .975 & .984 & .055 & & -.063 & $4-7$ & 61.46 & 7)*** & -.007 & -.002 & +.005 \\
\hline & & $4-9$ & Latent mean invariance & 812.84 & $79)^{* * *}$ & .887 & .931 & .114 & & -.121 & $4-8$ & 721.1 & $(7)^{* * * *}$ & -.088 & -.053 & +.059 \\
\hline Questionnaires & Models & & & $\mathrm{N}^{\circ}$ & Description & $\chi^{2}(\mathrm{df}$ & & CFI & TLI & RMSEA & $\begin{array}{l}\text { RMSEA } \\
90 \% \text { CI }\end{array}$ & $\mathrm{CM}$ & $\Delta \mathrm{R} \chi^{2}(\mathrm{df})$ & $\Delta \mathrm{CFI}$ & $\Delta$ TLI & $\triangle$ RMSEA \\
\hline WBIS-M & $\mathrm{M}$ & & & $5-1$ & $\begin{array}{l}\text { Configural } \\
\text { invariance }\end{array}$ & 1515 & $55(240)^{* * * *}$ & .952 & .928 & .086 & $.082-.090$ & - & - & - & - & - \\
\hline & & & & $5-2$ & $\begin{array}{l}\text { Weak } \\
\text { invariance }\end{array}$ & 2120 & $1(303)^{* * * *}$ & .931 & .918 & .091 & $.087-.095$ & $5-1$ & $635.49(63)^{* * *}$ & -.021 & -.010 & +.005 \\
\hline & & & & $5-3$ & $\begin{array}{l}\text { Partial } \\
\text { weak } \\
\text { invariance }\end{array}$ & 1757. & $37(292)^{* * * *}$ & .945 & .932 & .083 & $.080-.087$ & $5-1$ & $218.55(52)^{* * * *}$ & -.007 & +.004 & -.003 \\
\hline & & & & $5-4$ & $\begin{array}{l}\text { Strong } \\
\text { invariance }\end{array}$ & 2618. & $26(355)^{* * * *}$ & .914 & .913 & .094 & $.091-.097$ & $5-3$ & $1048.02(63)^{* * *}$ & -.031 & -.019 & +.011 \\
\hline & & & & $5-5$ & $\begin{array}{l}\text { Partial } \\
\text { strong } \\
\text { invariance }\end{array}$ & 1926. & $32(329)^{* * * *}$ & .940 & .934 & .082 & $.078-.086$ & $5-3$ & $153.78(37)^{* * *}$ & -.004 & +.002 & -.001 \\
\hline
\end{tabular}




\begin{tabular}{|c|c|c|c|c|c|c|c|c|c|c|}
\hline & & $5-6$ & Strict invariance & $2139.076(399)^{* * * *}$ & .934 & .941 & .078 & $.074-.081$ & $5-5$ & $262.38(70)^{\prime \prime}$ \\
\hline & & $5-7$ & CU invariance & $2240.555(434) * * * *$ & .932 & .943 & .076 & $.073-.079$ & $5-6$ & $135.84(35)^{*}$ \\
\hline & & $5-8$ & $\begin{array}{l}\text { Variance } \\
\text { invariance }\end{array}$ & $2334.302(441)^{* * * *}$ & .928 & .942 & .077 & $.074-.080$ & $5-7$ & $149.87(7)^{* *}$ \\
\hline & & $5-9$ & $\begin{array}{l}\text { Latent mean } \\
\text { invariance }\end{array}$ & $2561.892(448)^{* * *}$ & .920 & .936 & .081 & $.078-.084$ & $5-8$ & $406.14(7)^{* *}$ \\
\hline \multirow[t]{5}{*}{ BAS-2 } & DIF: Age, BMI, sex, and SES ( $n=5834)$ & $6-1$ & $\begin{array}{l}\text { MIMIC Null } \\
\text { effects }\end{array}$ & $4256.868(781)^{* * *}$ & .910 & .922 & .078 & $.076-.080$ & - & - \\
\hline & & $6-2$ & $\begin{array}{l}\text { MIMIC } \\
\text { Saturated }\end{array}$ & $2496.595(461)^{* * * *}$ & .947 & .922 & .078 & $.075-.081$ & $6-1$ & $917.80(320$ \\
\hline & & $6-3$ & $\begin{array}{l}\text { MIMIC Factor } \\
\text { only }\end{array}$ & $3708.022(749)^{* * *}$ & .923 & .930 & .074 & $.071-.076$ & $6-1$ & $313.67(32)^{*}$ \\
\hline & & $6-4$ & $\begin{array}{l}\text { MIMIC Factor } \\
\text { only - partial }\end{array}$ & $3106.504(703)^{* * * *}$ & .938 & .940 & .068 & $.066-.071$ & $6-2$ & $259.85(242$ \\
\hline & & $6-5$ & $\begin{array}{l}\text { MIMIC Factor } \\
\text { only - partial } \\
\text { (invariance) }\end{array}$ & $3220.946(731)^{* * * *}$ & .935 & .940 & .068 & $.066-.071$ & $6-4$ & $59.37(28)^{* * *}$ \\
\hline \multirow[t]{4}{*}{ BASS } & DIF: Age, BMI, sex, and SES ( $n=5925)$ & $7-1$ & $\begin{array}{l}\text { MIMIC Null } \\
\text { effects }\end{array}$ & $3907.411(648)^{* * * *}$ & .828 & .847 & .082 & $.080-.085$ & - & - \\
\hline & & $7-2$ & $\begin{array}{l}\text { MIMIC } \\
\text { Saturated }\end{array}$ & $1281.664(360)^{* * *}$ & .951 & .922 & .059 & $.055-.062$ & $7-1$ & $1399.70(28$ \\
\hline & & $7-3$ & $\begin{array}{l}\text { MIMIC Factor } \\
\text { only }\end{array}$ & $3085.276(616)^{* * *}$ & .870 & .878 & .074 & $.071-.076$ & $7-1$ & $445.66(32)^{\prime}$ \\
\hline & & $7-5$ & $\begin{array}{l}\text { MIMIC Factor } \\
\text { only - partial } \\
\text { (invariance) }\end{array}$ & $1714.772(562)^{* * *}$ & .939 & .938 & .053 & $.050-.055$ & $7-4$ & $52.92(28)^{* * *}$ \\
\hline \multirow[t]{5}{*}{ PACS } & DIF: Age, BMI, sex, and SES ( $n=5645)$ & $8-1$ & $\begin{array}{l}\text { MIMIC Null } \\
\text { effects }\end{array}$ & $1128.109(200)^{* * *}$ & .901 & .913 & .081 & $.077-.086$ & - & - \\
\hline & & $8-2$ & $\begin{array}{l}\text { MIMIC } \\
\text { Saturated }\end{array}$ & $212.596(72)^{* * *}$ & .985 & .963 & .053 & $.045-.061$ & $8-1$ & $496.20(128$ \\
\hline & & $8-3$ & $\begin{array}{l}\text { MIMIC Factor } \\
\text { only }\end{array}$ & $678.714(168)^{* * *}$ & .946 & .943 & .066 & $.061-.071$ & $8-1$ & $245.36(32)^{\prime}$ \\
\hline & & $8-4$ & $\begin{array}{l}\text { MIMIC Factor } \\
\text { only - partial }\end{array}$ & $317.070(146)^{* * *}$ & .982 & .978 & .041 & $.035-.047$ & $8-2$ & $46.76(74)$ \\
\hline & & $8-5$ & $\begin{array}{l}\text { MIMIC Factor } \\
\text { only - partial } \\
\text { (invariance) }\end{array}$ & $423.707(174)^{* * * *}$ & .973 & .973 & .045 & $.040-.051$ & $8-4$ & $56.76(28)^{* * *}$ \\
\hline \multirow[t]{3}{*}{ WBIS-M } & DIF: Age, BMI, sex, and SES ( $n=5664)$ & $9-1$ & $\begin{array}{l}\text { MIMIC Null } \\
\text { effects }\end{array}$ & $4392.168(768)^{* * *}$ & .890 & .903 & .082 & $.079-.084$ & - & - \\
\hline & & $9-2$ & $\begin{array}{l}\text { MIMIC } \\
\text { Saturated }\end{array}$ & $2235.517(448)^{* * * *}$ & .946 & .918 & .075 & $.072-.078$ & $9-1$ & $1230.99(32$ \\
\hline & & $9-3$ & $\begin{array}{l}\text { MIMIC Factor } \\
\text { only }\end{array}$ & $3344.924(736)^{* * * *}$ & .921 & .927 & .071 & $.068-.073$ & $9-1$ & $691.98(32)^{4}$ \\
\hline
\end{tabular}




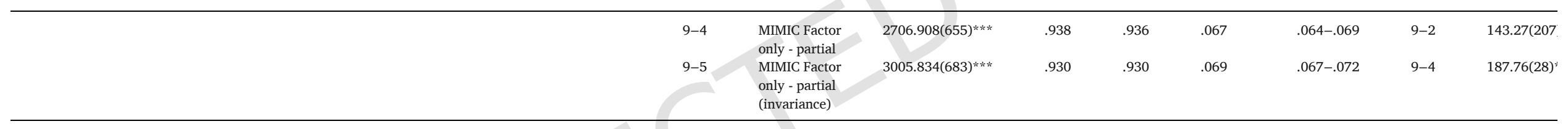

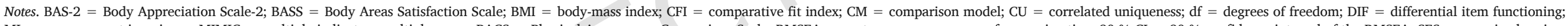

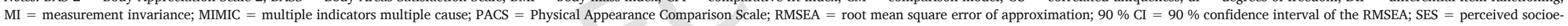

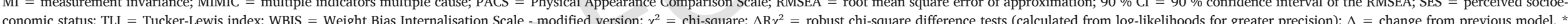

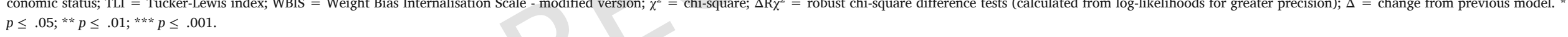


Table 2

Standardized Parameters Estimates from the Confirmatory Factor Analyses of the BAS-2, BASS, PACS and WBIS-M in the Overall Sample.

\begin{tabular}{|c|c|c|c|c|}
\hline Questionnaires & Items & $\lambda$ & $\delta$ & $\omega$ \\
\hline \multirow[t]{10}{*}{ BAS-2 } & BAS-2_1 & .776 & .398 & .952 \\
\hline & BAS-2_2 & .868 & .247 & \\
\hline & BAS-2_3 & .786 & .383 & \\
\hline & BAS-2_4 & .896 & .197 & \\
\hline & BAS-2_5 & .717 & .486 & \\
\hline & BAS-2_6 & .859 & .262 & \\
\hline & BAS-2_7 & .808 & .347 & \\
\hline & BAS-2_8 & .775 & .399 & \\
\hline & BAS-2_9 & .869 & .244 & \\
\hline & BAS-2_10 & .793 & .370 & \\
\hline \multirow[t]{9}{*}{ BASS } & BASS1 & .528 & .722 & .852 \\
\hline & BASS2 & .390 & .848 & \\
\hline & BASS3 & .712 & .492 & \\
\hline & BASS4 & .703 & .506 & \\
\hline & BASS5 & .675 & .544 & \\
\hline & BASS6 & .641 & .589 & \\
\hline & BASS7 & .724 & .475 & \\
\hline & BASS8 & .363 & .868 & \\
\hline & BASS9 & .821 & .327 & \\
\hline \multirow[t]{4}{*}{ PACS } & PACS1 & .898 & .194 & .853 \\
\hline & PACS2 & .467 & .782 & \\
\hline & PACS3 & .777 & .396 & \\
\hline & PACS5 & .887 & .214 & \\
\hline \multirow[t]{19}{*}{ WBIS-M } & WBIS- & .794 & .369 & .943 \\
\hline & M_2 & & & \\
\hline & $\begin{array}{l}\text { WBIS- } \\
\text { M } 3\end{array}$ & .825 & .320 & \\
\hline & WBIS- & .850 & .277 & \\
\hline & M_4 & & & \\
\hline & WBIS- & .906 & .180 & \\
\hline & M_5 & & & \\
\hline & WBIS- & .827 & .316 & \\
\hline & M_6 & & & \\
\hline & WBIS- & .771 & .406 & \\
\hline & M_7 & & & \\
\hline & WBIS- & .672 & .548 & \\
\hline & M_8 & & & \\
\hline & WBIS- & .625 & .609 & \\
\hline & M_9 & & & \\
\hline & WBIS- & .784 & .385 & \\
\hline & M_10 & & & \\
\hline & WBIS- & .818 & .331 & \\
\hline & M_11 & & & \\
\hline
\end{tabular}

Notes. BAS-2 $=$ Body Appreciation Scale-2; BASS $=$ Body Areas Satisfaction Scale; PACS $=$ Physical Appearance Comparison Scale; WBIS $=$ Weight Bias Internalization Scale - modified version; $\lambda=$ factor loadings; $\delta=$ Uniquenesses; $\omega=$ McDonald's omega coefficient of composite reliability.

to .384). Finally, as presented in Table 2 , the composite reliability of the BASS was good.

\subsubsection{PACS}

The a priori one-factor CFA resulted in an acceptable (for RMSEA) to good (for CFI/TLI) level of fit to the data (see model 1-4). However, inspection of the parameters revealed that Item 4 ("comparing your 'looks' to the 'looks' of others is a bad way to determine if you are attractive or unattractive") had a suboptimal standardized loading ( $\lambda=$ .149). Therefore, this item was excluded, and the model was re-examined. The resulting model presented in Table 1 (see model 1-5) provided a perfect fit to the data. The standardized parameter estimates from this model, which are presented in Table 2, revealed that all loadings are substantial $\left(M_{\lambda}=.757\right.$; ranging from .467 to .898$)$. Finally, the composite reliability of the PACS was good (see Table 2).

\subsubsection{WBIS-M}

As presented in Table 1 (see model 1-6), the a priori one-factor CFA resulted in a poor (for all indices) level of fit to the data. In order to improve the $a$ priori model, the parameter estimates, and modification indices provided by Mplus were inspected. First, analysis of the standardized parameter estimates revealed that Item 1 (a reverse-coded item: 'Because of my weight, I feel that I am just as competent as anyone') was associated with a suboptimal standardized loading $(\lambda=.229)$. Second, to improve the model, the modification indices suggested correlating the residual variances of Items 7 ("My weight is a major way that I judge my value as a person") and 6 ("I hate myself for my weight"), 6 ("I hate myself for my weight") and 5 ("Whenever I think about my weight, I feel depressed"), 3 ("I feel anxious about my weight because of what people might think of me") and 2 ("I am less attractive than most other people because of my weight"), 8 ("I don't feel that I deserve to have a really fulfilling social life because of my weight") and 6 ("I hate myself for my weight"), and 8 ("I don't feel that I deserve to have a really fulfilling social life because of my weight") and 7 ("My weight is a major way that I judge my value as a person"). This alternative model (see model 1-7) comprising 10 items (instead of 11) and five correlated residual variances resulted in a substantial improvement of model fit indices and provided an acceptable (for RMSEA and TLI) to good (for CFI) level of fit to the data (see Table 1). The standardized parameter estimates from this model, which are presented in Table 2, show that all loadings are substantial $\left(M_{\lambda}=.787\right.$; ranging from .625 to .906$)$. The correlation between the residual variances are significant (at $p<.001$ ), positive and modest ( $M=.306$; ranging from .254 to .406 ). Finally, as presented in Table 2, the WBIS-M revealed an excellent composite reliability.

\subsection{Measurement invariance of the factor structure of the BAS-2, BASS, $P A C S$, and WVIS-M across countries}

The goodness-of-fit statistics of the measurement invariance of the factor structure of the BASS, BAS-2, WBIS-M, and PACS across countries, are presented in Table 1 (see models $2-1$ to 5-9).

\subsubsection{BAS-2 and BASS}

Tests of measurement invariance of the a priori factor structure of the BAS- 2 and alternative factor structure of the BASS conducted across countries (see models $2-1 / 3-1$ to $2-8 / 3-9$ ) revealed that the CFI, TLI, and RMSEA indicated acceptable to good model fit at all steps. More specifically, results provided support for a weak invariance (loadings; see models $2-2 / 3-2 ; \Delta$ CFIs/TLI $\leq .01$ and $\triangle$ RMSEAs $\leq .015$ ), but a lack of strong (intercepts; see models $2-3 / 3-3 ; \Delta$ CFIs/TLIs $\geq .01$ and $\Delta$ RMSEAs $\geq .015$ ) and strict invariance (uniqueness; see models $2-5 / 3-5$; $\Delta$ CFIs $\geq .01$ ).

Analysis of modification indices revealed for the: (a) BAS-2 that 15 item intercepts and 7 item uniqueness (on a possibility of 80) and; (b) BASS that 19 item intercepts and 16 item uniqueness (on a possibility of 72); are not invariant across countries (see Table S1 in the supplementary file for more details on the items and countries). Therefore, the equality constraints on these item intercepts and uniqueness were allowed to be freely estimated in some countries (see Table S1), and the models were re-estimated. Their results provide support for the partial strong (see models $2-4 / 3-4$; CFIs/TLIs $\leq .01$ and $\triangle$ RMSEAs $\leq .015$ ) and strict (see models 2-6/3-6; CFIs/TLIs $\leq .01$ and $\triangle$ RMSEAs $\leq .015$ ) invariance of the BAS- 2 and BASS across countries. An additional step for the BASS provided support for the invariance of the correlated residual variances across countries (see model $3-7 ; \mathrm{CFI} / \mathrm{TLI} \leq .01$ and $\triangle$ RMSEA $\leq .015)$. 
Finally, the last two steps of measurement invariance tests not related to the invariance (or lack of) of the measurement model itself, but rather related to cross-country comparisons provided support for the invariance of latent variance (see models $2-7 / 3-8$; CFIs/TLIs $\leq .01$ and $\triangle$ RMSEAs $\leq$. 015), but a lack of latent mean invariance (see models 2-8/3-9; $\Delta$ CFIs/TLIs $\geq .01$ ), indicating country differences in the latent constructs of the BAS- 2 and BASS. Significant latent mean differences for the BAS-2 and BASS across the countries are presented in Table 3 .

\subsubsection{PACS and WBIS-M}

Tests of measurement invariance of the alternative factor structures of the PACS and WBIS-M conducted across countries (see models 4-1/ 5-1 to 4-9/5-9) revealed that the CFI, TLI, and RMSEA indicated acceptable to good model fit at all steps. More specifically, results not only revealed a lack of weak invariance (loadings; see models 4-2/ 5-2; PACS: $\Delta$ CFI/TLI $\geq .01$ and $\Delta$ RMSEA $\geq .015$; WBIS-M: $\Delta$ CFI $\geq .01$ ) and strong invariance (intercepts; see model 4-4/5-4; PACS: $\triangle \mathrm{CFI}$ TLI $\geq .01$ and $\triangle$ RMSEAs $\geq .015$; WBIS-M: $\Delta$ CFI/TLI $\geq .01$ ) for both the WBIS-M and PACS, but also a lack of strict invariance for the PACS (uniqueness; see model 4-6; $\Delta$ CFI/TLI $\geq .01$ and $\triangle$ RMSEA $\geq .015$ ).

Analysis of modification indices revealed for the: (a) PACS that 4 item loadings, 7 item intercepts and 10 item uniqueness (on a possibility of 32); and (b) WBIS-M that 11 item loadings and 26 item intercepts (on a possibility of 80) are not invariant across countries (see the Table S1). Therefore, the equality constraints on these item loadings, intercepts, and uniquenesses were allowed to be freely estimated in some countries (see Table S1), and the models were re-estimated. The results provided support for the partial weak (see models 4-3/5-3; CFIs/TLIs $\leq .01$ and $\triangle$ RMSEAs $\leq .015$ ) and strong (see models $4-5 / 5-5$; CFIs/TLIs $\leq .01$ and $\triangle$ RMSEAs $\leq .015$ ) invariance of the WBIS-M and PACS across countries, as well as the partial strict (see model $4-7$; CFI/TLI $\leq .01$ and $\triangle$ RMSEA $\leq$.015) invariance of the PACS across countries. An additional step for the WBIS-M provided support for the invariance of the correlated residual variances across countries (see model 5-7; CFI/TLI $\leq .01$ and $\triangle R M-$ $\mathrm{SEA} \leq$.015).

Finally, the last two steps not only provided support for an invariance of latent variance for both the PACS and WBIS-M (see models 4-8/ $5-8$; CFIs/TLIs $\leq .01$ and $\triangle$ RMSEAs $\leq .015$ ), but also either a lack of latent mean invariance for the WBIS-M (see model 5-9; $\Delta$ CFI/TLI $\leq .01$ and $\triangle$ RMSEAs $\leq .015$ ) and a latent mean invariance for the PACS (see model $4-9 ; \Delta$ CFI/TLI $\geq .01$ and $\triangle$ RMSEA $\geq .015$ ). Significant latent mean differences for the PACS across the countries are presented in Table 3.

\subsection{DIF and latent mean differences: age, BMI, sex and SES}

The goodness-of-fit statistics of the MIMIC models of the BAS-2, BASS, PACS, and WBIS-M across countries are displayed in Table 1 (see models 6-1 to 9-5). As mentioned in the analyses section, these models were estimated starting from the most invariant measurement model (see models 2-7, 3-8, and 4-8: invariance of the latent variance for the BAS-2, BASS, and PACS, respectively; 5-9: latent mean invariance for the WBIS-M). Results first show that the null effects model (see models 6-1, 7-1, 8-1, 9-1) resulted in acceptable fit indices for the BAS-2 and the PACS and poor fit indices for the BASS and WBIS-M. Additional results showed that both the saturated (see models 6-2, 7-2, 8-2, 9-2; BAS-2/WBIS-M: $\triangle$ CFIs $\geq .01$; BASS/PACS: $\triangle$ CFIs/TLIs $\geq .01$ and $\triangle$ RMSEAs $\geq .015$ ) and the factor only (see models $6-3,7-3$, 8-3, 9-3; BAS-2: $\triangle$ CFI $\geq .01$; PACS: CFI/TLI $\geq .01$ and $\triangle$ RMSEA $\geq$ .015 ; BASS/WBIS-M: $\triangle$ CFIs/TLIs $\geq .01$ ) models resulted in a substantial improvement in model fit compared to the null effects model. These findings support the idea that the demographic predictors are signifi-
Table 3

Latent Mean Differences Observed for the Scales of the BAS-2, BASS, and PACS Across Countries.

\begin{tabular}{|c|c|c|c|}
\hline Questionnaires & Countries & $\begin{array}{l}\text { Significantly lower } \\
\text { latent factor mean } \\
\text { than }\end{array}$ & $\begin{array}{l}\text { Significantly higher } \\
\text { latent factor mean } \\
\text { than }\end{array}$ \\
\hline \multirow[t]{8}{*}{ BAS-2 } & $\begin{array}{l}\text { Australia ( } n \\
=555)\end{array}$ & $\begin{array}{l}\text { Belgium, Canada, } \\
\text { China, Italy, Spain, } \\
\text { USA }\end{array}$ & Japan \\
\hline & $\begin{array}{l}\text { Belgium ( } n \\
=593)\end{array}$ & China, Spain & $\begin{array}{l}\text { Australia, Canada, } \\
\text { Japan, USA }\end{array}$ \\
\hline & $\begin{array}{l}\text { Canada }(n \\
=715)\end{array}$ & $\begin{array}{l}\text { Belgium, China, Italy, } \\
\text { Spain, USA }\end{array}$ & Australia, Japan \\
\hline & $\begin{array}{l}\text { China }(n= \\
614)\end{array}$ & Spain & $\begin{array}{l}\text { Australia, Belgium, } \\
\text { Canada, Italy, Japan, } \\
\text { USA }\end{array}$ \\
\hline & $\begin{array}{l}\text { Italy }(n= \\
620)\end{array}$ & China, Spain & $\begin{array}{l}\text { Australia, Canada, } \\
\text { Japan }\end{array}$ \\
\hline & $\begin{array}{l}\text { Japan }(n= \\
601)\end{array}$ & $\begin{array}{l}\text { Australia, Belgium, } \\
\text { Canada, China, Italy, }\end{array}$ & \\
\hline & $\begin{array}{l}\text { Spain }(n= \\
814)\end{array}$ & & $\begin{array}{l}\text { Australia, Belgium, } \\
\text { Canada, China, Italy, } \\
\text { Japan, USA }\end{array}$ \\
\hline & $\begin{array}{l}\text { USA }(n= \\
1443)\end{array}$ & Belgium, China, Spain & $\begin{array}{l}\text { Australia, Canada, } \\
\text { Japan }\end{array}$ \\
\hline \multirow[t]{8}{*}{ BASS } & $\begin{array}{l}\text { Australia ( } n \\
=566)\end{array}$ & $\begin{array}{l}\text { Belgium, Canada, } \\
\text { China, Italy, Spain, } \\
\text { USA }\end{array}$ & Japan \\
\hline & $\begin{array}{l}\text { Belgium ( } n \\
=601)\end{array}$ & Spain & $\begin{array}{l}\text { Australia, Canada, } \\
\text { China, Japan, USA }\end{array}$ \\
\hline & $\begin{array}{l}\text { Canada }(n \\
=731)\end{array}$ & Belgium, Italy, Spain & Australia, Japan \\
\hline & $\begin{array}{l}\text { China }(n= \\
625)\end{array}$ & Belgium, Italy, Spain & Australia, Japan \\
\hline & $\begin{array}{l}\text { Italy }(n= \\
629)\end{array}$ & Spain & $\begin{array}{l}\text { Australia, Canada, } \\
\text { China, Japan, USA }\end{array}$ \\
\hline & $\begin{array}{l}\text { Japan }(n= \\
612)\end{array}$ & $\begin{array}{l}\text { Australia, Belgium, } \\
\text { Canada, China, Italy, } \\
\text { Spain, USA }\end{array}$ & \\
\hline & $\begin{array}{l}\text { Spain }(n= \\
817)\end{array}$ & & $\begin{array}{l}\text { Australia, Belgium, } \\
\text { Canada, China, Italy, } \\
\text { Japan, USA }\end{array}$ \\
\hline & $\begin{array}{l}\text { USA }(n= \\
1465)\end{array}$ & Italy, Belgium, Spain & Australia, Japan \\
\hline \multirow[t]{8}{*}{ PACS } & $\begin{array}{l}\text { Australia ( } n \\
=524)\end{array}$ & & $\begin{array}{l}\text { Belgium, Canada, } \\
\text { China, Italy, Japan, } \\
\text { Spain, USA }\end{array}$ \\
\hline & $\begin{array}{l}\text { Belgium ( } n \\
=571)\end{array}$ & Australia, Canada & China, Italy, Spain \\
\hline & $\begin{array}{l}\text { Canada ( } n \\
=667)\end{array}$ & Australia & $\begin{array}{l}\text { Belgium, China, Italy, } \\
\text { Spain }\end{array}$ \\
\hline & $\begin{array}{l}\text { China }(n= \\
601)\end{array}$ & $\begin{array}{l}\text { Australia, Belgium, } \\
\text { Canada, Japan, USA }\end{array}$ & Spain \\
\hline & $\begin{array}{l}\text { Italy }(n= \\
593)\end{array}$ & $\begin{array}{l}\text { Australia, Belgium, } \\
\text { Canada, Japan, USA }\end{array}$ & China, Spain \\
\hline & $\begin{array}{l}\text { Japan }(n= \\
594)\end{array}$ & Australia & $\begin{array}{l}\text { Belgium, China, Italy, } \\
\text { Spain }\end{array}$ \\
\hline & $\begin{array}{l}\text { Spain }(n= \\
809)\end{array}$ & $\begin{array}{l}\text { Australia, Belgium, } \\
\text { Canada, China, Italy, } \\
\text { Japan, USA }\end{array}$ & \\
\hline & $\begin{array}{l}\text { USA }(n= \\
1404)\end{array}$ & Australia & $\begin{array}{l}\text { Belgium, China, Italy, } \\
\text { Spain }\end{array}$ \\
\hline
\end{tabular}

Notes. BAS-2 = Body Appreciation Scale-2; BASS = Body Areas Satisfaction Scale; PACS $=$ Physical Appearance Comparison Scale; USA = United States of America.

cantly associated with BAS-2, BASS, PACS, and WBIS-M responses. However, the factor only model resulted in substantially lower model fit than the saturated model (BASS: $\Delta \mathrm{R} \chi^{2}=1399.70$, df $=288, p<$ $.001, \Delta \mathrm{CFI}=+.123, \Delta \mathrm{TLI}=+.075, \Delta \mathrm{RMSEA}=-.023 ; \mathrm{BAS}-2: \Delta \mathrm{R} \chi^{2}$ 
$=917.80, \mathrm{df}=320, p<.001, \Delta \mathrm{CFI}=+.037, \Delta \mathrm{TLI}=.000, \Delta \mathrm{RM}-$ SEA $=.000$; WBIS-M: $\Delta \mathrm{R} \chi^{2}=1230.99, \mathrm{df}=320, p<.001, \Delta \mathrm{CFI}$ $=+.056, \Delta \mathrm{TLI}=+.015, \Delta \mathrm{RMSEA}=-.007$; PACS: $\Delta \mathrm{R} \chi^{2}=496.20$, $\mathrm{df}=128, p<.001, \Delta \mathrm{CFI}=+.084, \Delta \mathrm{TLI}=+.050, \Delta$ RMSEA $=$ -.028), revealing DIF as a function of the demographic predictors. Examination of the modification indices of the model suggested that DIF might be attributed to several items and predictors in some countries (see Table S2 for more details on the items, the predictors and the countries). Therefore, a fourth model of partial factor only invariance was estimated by freeing several paths from the demographic predictors to the item responses in some countries (see Table S2 for more details). These models that resulted in an acceptable level of fit to the data (see models $6-4,7-4,8-4,9-4)$ do not show a substantial decrease of fit indices compared to the saturated model ( $\Delta$ CFIs/RMSEAs $\leq .01 / .015$ and $\Delta$ TLIs $\geq .01$ ).

Finally, in the last model (see models $6-5,7-5,8-5,9-5$ ), starting with the factor only partial model, the relationships between the demographic predictors and the latent factors of the BAS-2, BASS, WBIS-M, and PACS were constrained to be equal across countries. Results showed that changes in fit indices were not substantial ( $\Delta$ CFIs/TLIs $\leq .01$ and $\triangle$ RMSEAs $\leq .015$ ), supporting the equivalence of the relationship between the demographic predictors and the BAS-2, BASS, PACS and WBIS-M ratings across countries.

The results from these final models are presented in Table 4, where it can be seen that sex and BMI significantly and negatively predict the latent factors of the BAS-2 and BASS, whereas perceived SES significantly and positively predicts the latent factors of the BAS-2 and BASS. This means that women (compared to men) and individuals with higher BMIs (compared to those with lower BMIs) tended to present lower levels of body satisfaction and appreciation, whereas individuals with higher perceived SES (compared to those with lower perceived SES) tended to have higher levels of body satisfaction and appreciation. Age was also predictive of BAS-2 scores, such that older participants (compared to younger) tended to have higher levels of body appreciation.
Further, the results showed that sex and BMI significantly and positively predict the latent factors of the PACS and WBIS-M, whereas perceived SES significantly and negatively predicts the latent factors of the PACS and WBIS-M (Table 4). This means that women (compared to men) and individuals with higher BMIs (compared to those with lower BMIs) tended to present higher levels of weight bias internalization and physical appearance comparison; whereas individuals with higher perceived SES (compared to those with lower perceived SES) tended to have lower levels of weight bias internalization and physical appearance comparison.

\section{Discussion}

This study's aim was to assess the measurement invariance of two measures of positive body image and two measures of constructs related to positive body image (e.g. physical appearance comparison and weight bias), across eight countries and seven different languages. The present findings are important given that few studies have specifically focused on possible cross-country differences in positive body image within the same study (Tiggemann, 2015) and no study so far has used the BAS-2, BASS, PACS, and WBIS-M to assess possible similarities and differences in body satisfaction, body appreciation, physical appearance comparison and weight bias across countries and different languages. Overall, the findings show that the adjusted models of the four different measures were a good fit to the data. Our results also indicate partial factorial invariance of the four measures in a sample of emerging adults aged 18-30. Although some differences were noted in the item loadings, intercept, and uniqueness, as well as in the latent constructs of these four measures, the vast majority of items worked well across countries. Moreover, the MIMIC models support the equivalence of the relationship between the demographic predictors. The results also suggest that sociodemographic characteristics such as gender, BMI, and socioeconomic status contribute to differences across countries and so these should be considered (and possibly controlled for) when using these questionnaires in comparative research. Thus, the

Table 4

Unstandardized and Standardized Relations between the BAS-2, BASS, PACS, WBIS-M and the Predictors.

\begin{tabular}{|c|c|c|c|c|c|c|c|c|c|c|}
\hline & & & Sample-spe & tandardized c & ficients. & & & & & \\
\hline Questionnaires & Predictors & $b$ (SE) & $\beta$ (Australia) & $\beta$ (Belgium) & $\beta$ (Canada) & $\beta$ (China) & $\beta$ (Italy) & $\beta$ (Japan) & $\beta$ (Spain) & $\beta$ (USA) \\
\hline \multirow[t]{4}{*}{ BAS-2 } & Age & $.034(.014)^{*}$ & $.032^{*}$ & $.032^{*}$ & $.032 *$ & $.032^{*}$ & $.032 *$ & $.032^{*}$ & $.032^{*}$ & $.032 *$ \\
\hline & BMI & $-.227(.015)^{* * *}$ & $-.217 * * *$ & $-.217^{* * *}$ & $-.218^{*}$ & $-.217^{* * *}$ & $-.219^{* * *}$ & $-.218^{* * *}$ & $-.218^{* * *}$ & $-.216^{* * *}$ \\
\hline & Sex & $-.382(.031)^{* * * *}$ & $-.171^{* * *}$ & $-.176^{* * *}$ & $-.143^{* * *}$ & $-.182^{* * *}$ & $-.163^{* * *}$ & $-.131^{* * *}$ & $-.180 * * *$ & $-.168^{* * *}$ \\
\hline & SES & $.127(.014)^{* * *}$ & $.121^{* * *}$ & $.121^{* * *}$ & $.122^{* * *}$ & $.122^{* * *}$ & $.123^{* * *}$ & $.122^{* * *}$ & $.122^{* * *}$ & $.121^{* * *}$ \\
\hline \multirow[t]{4}{*}{ BASS } & Age & $.023(.014)$ & .022 & .022 & .022 & .022 & .022 & .022 & .022 & .022 \\
\hline & BMI & $-.257(.018)^{* * *}$ & $-.241 * * *$ & $-.242^{* * *}$ & $-.244 *$ & $-.242^{* * *}$ & $-.245^{* * *}$ & $-.244^{* * *}$ & $-.243^{* * *}$ & $-.240^{* * *}$ \\
\hline & Sex & $-.465(.033)^{* * *}$ & $-.206^{* * *}$ & $-.211^{* * *}$ & $-.173^{* * *}$ & $-.218^{* * *}$ & $-.195^{* * *}$ & $-.160^{* * *}$ & $-.216^{* * *}$ & $-.201^{* * *}$ \\
\hline & SES & $.141(.015)^{* * *}$ & $.132^{* * *}$ & $.133^{* * *}$ & $.134^{* * *}$ & $.133^{* * *}$ & $.135^{* * *}$ & $.134^{* * *}$ & $.133^{* * *}$ & $.132^{* * *}$ \\
\hline \multirow[t]{4}{*}{ PACS } & Age & $-.004(.014)$ & -.004 & -.004 & -.004 & -.004 & -.004 & -.004 & -.004 & -.004 \\
\hline & BMI & $.110(.016)^{* * *}$ & $.105^{* * *}$ & $.105^{* * *}$ & $.106^{* * *}$ & $.105^{* * *}$ & $.106^{* * *}$ & $.107^{* * *}$ & $.105^{* * *}$ & $.105^{* * *}$ \\
\hline & Sex & $.648(.033)^{* * * *}$ & $.290 * * *$ & $.297 * * *$ & $.245^{* * *}$ & $.306^{* * *}$ & $.278^{* * *}$ & $.225^{* * *}$ & $.304^{* * *}$ & $.285^{* * *}$ \\
\hline & SES & $-.007(.015)$ & -.007 & -.007 & -.007 & -.007 & -.007 & -.007 & -.007 & -.007 \\
\hline \multirow[t]{4}{*}{ WBIS-M } & Age & $-.028(.014)$ & -0.025 & -0.025 & -0.025 & -0.025 & -0.025 & -0.025 & -0.025 & $-0.025^{*}$ \\
\hline & BMI & $.413(.018)^{* * * *}$ & $0.375^{* * *}$ & $0.378^{* * *}$ & $0.378 * * *$ & $0.376^{* * *}$ & $0.382^{* * *}$ & $0.378^{* * *}$ & $0.377^{* * *}$ & $0.374^{* * *}$ \\
\hline & Sex & $.463(.030)^{* * *}$ & $0.198^{* * *}$ & $0.203^{* * *}$ & $0.166^{* * *}$ & $0.210^{* * *}$ & $0.190^{* * *}$ & $0.152^{* * *}$ & $0.208^{* * *}$ & $0.193^{* * *}$ \\
\hline & SES & $-.052(.015)^{* * *}$ & $-0.047^{* * *}$ & $-0.047^{* * *}$ & $-0.047^{* * *}$ & $-0.047^{* * *}$ & $-0.048^{* * *}$ & $-0.047^{* * *}$ & $-0.047^{* * *}$ & $-0.047^{* * *}$ \\
\hline
\end{tabular}

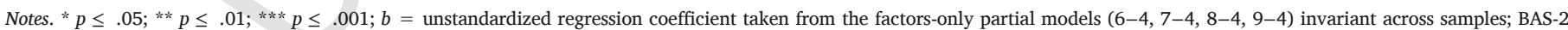

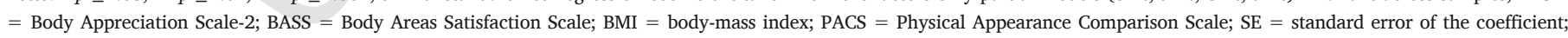

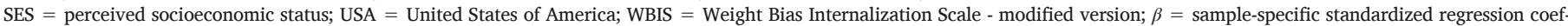

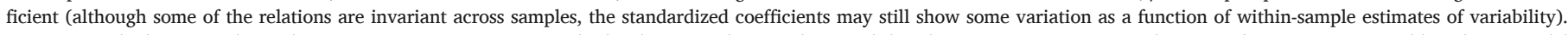

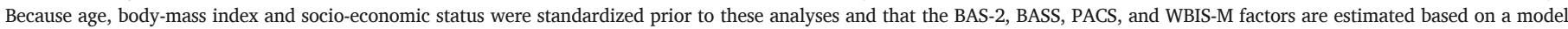
of latent variance invariance in which all latent factors have a $S D$ of 1 , all unstandardized coefficients can be directly interpreted in $S D$ units. 
BAS-2, BASS, PACS, and WBIS-M can be used to make valid comparisons across countries.

\subsection{Support for the cross-validity of the scales}

Significant improvements were obtained for the BASS and the WBIS-M by correlating residual variances. The BAS-2 did not require modification to reach a good model fit. In all cases, some items' intercepts and uniqueness were not invariant across countries and free estimation was necessary to improve the measurement invariance of the four scales. Taken together, these results suggest partial invariance of the BAS-2, BASS, PACS, and WBIS-M. Thus, it appears that respondents from Australia, Belgium, Canada, China, Italy, Japan, Spain, and the United States may interpret these four measures in a similar manner.

Our results suggest that the internal consistency of the four scales is good to excellent and that the BAS-2, BASS, PACS, and WBIS-M are reliable measures to use. Moreover, the constructs to which they refer seem to have relatively the same meaning to participants across our eight participating countries. The similarities that we found may be explained by an increasing global idealization of thinness (Swami, 2015; Swami et al., 2010). In fact, the globalization of the thin ideal, through westernization and modernization has led some researchers to suggest that differences in body size ideals may not be salient anymore (Swami, 2015), with individuals living in Western sites having equivalent body size ideals as those living in urban non-Western sites. Our results suggest this globalization phenomenon may not be limited to the thin ideal, but could also include body appreciation and satisfaction, physical appearance comparison, and weight bias.

As pointed out by Tylka and Wood-Barcalow (2015a), some aspects of body appreciation like "feeling good about one's body, accepting one's body and its unique qualities, and taking a positive attitude toward one's body" (p. 127), could be similar across cultures and countries. Moreover, people living in socio-economically advantaged countries may tend to hold similar weight bias and anti-fat attitudes, for example thinking that higher weight is under one's personal control and mostly caused by unhealthy eating and exercising habits (Flegal, Kruszon-Moran, Carroll, Fryar, \& Ogden, 2016). They may also share a tendency to compare themselves to others, which could similarly affect their body image. Consistent with this, researchers found that while unflattering and upward comparison is associated with body dissatisfaction, efforts to avoid comparison could instead have a positive impact on body image (Fuller-Tyszkiewicz et al., 2019).

\subsection{Considerations for the use of these measures in cross-country comparisons}

As suggested by Swanson (2016), our results also imply that specificities across countries need to be considered. More specifically, in the case of emerging adults, some differences may be unique to their country of belonging. In fact, some of the items used to assess body satisfaction (i.e., satisfaction with different body parts), body appreciation (i.e., overall positive orientation towards the body), ability to filter information and reciprocity (i.e., weight bias and physical appearance comparison) seemed to slightly differ from one country to the other. These results are consistent with those of past research indicating that some components of positive body image are not perceived similarly across cultures. For example, other researchers have found that components such as adaptive appearance investment may be culture-specific (Tiggemann, 2015; Tylka \& Wood-Barcalow, 2015a). Moreover, as pointed out by Tiggemann (2015), some ethnical backgrounds (e.g., African and Hispanic) may convey a more positive body image and a broader conceptualization of beauty.

Cross-country comparisons of these scales should also take linguistic issues into account. Although we followed careful translation and back- translation procedures when preparing the questionnaires for this study, we must consider that the conceptualization and terms used to describe positive body image may be understood differently in the different countries that took part in this study (see Ng, Barron, \& Swami, 2015). As each language has distinct taxonomies and lexemes (Fiske, 2020), some items may not have a one-to-one correspondence in different languages. For example, with regards to the PACS, the word "looks" found in item 4 ( "comparing your "looks" to the "looks" of others is a bad way to determine if you are attractive or unattractive') could have contributed to the need to remove this item from the version used for cross-cultural comparisons. In fact, the word "looks", may not have a one-on-one equivalent in every language. This possibility seems even more probable in light of the fact that it was difficult to translate this word in the six non-English languages of this study. Thus, the translated words that were used couldn't exactly tap into the same concept as the English one, implying that non-English speakers were likely to understand these items in a different manner. Thus, some constructs should be reconsidered and challenged, as they are imperfect tools for identifying constructs (Fiske, 2020) and potential linguistic issues should be monitored when studying positive body image across samples from different countries (Tiggemann, 2015). In doing so, qualitative research focusing on the meaning of positive body image, weight bias and physical appearance comparison could help identify and further explore the words that specifically refer to these concepts across different countries and languages.

In the case of the WBIS-M, we had to remove item 1 , the only reversed item of this measure ('Because of my weight, I feel that I am just as competent as anyone'). While the suboptimal standardized loading of this item may reflect linguistic issues (for example with the translation of the word "feel"), it also suggests that, when using the WBIS-M for cross-cultural purposes and across different languages, it may be preferable to opt for a version exempt from reversed items and in which items are phrased in a similar way. Although we have removed items to simplify models and have reasons to believe that the constructs measured with the remaining items were not impacted, caution should still be taken before deciding that item deletion is the best approach to use. Future studies should consider either removing or rewording those items when using these questionnaires for comparative purposes.

Additionally, participants' recruitment could contribute to some cross-country differences. First, incentives for participation were given in some countries. Although, incentives were very modest and unlikely to be perceived as coercive, they may have facilitated recruitment and motivate participation of individuals less inclined to participate otherwise (Singer \& Bossarte, 2006), thus possibly contributing to some cross-country differences. Second, the age group of our participants (18-30 years old) corresponds to a developmental period of changes in terms of weight as well as eating, exercising, sleeping, and drinking habits (Aimé, Villatte, Cyr, \& Marcotte, 2017; Kelly \& Latner, 2015). Emerging adults tend to adopt suboptimal healthy habits and to gain weight, thus increasing their likelihood of being more preoccupied with their appearance. While all emerging adults are at risk of experiencing these changes, they are also likely to be influenced by social desirability and the social norms of their country of belonging (Swanson, 2016). Thus, appearance preoccupations may operate differently from one country to the other: while some participants from a given country may worry more about their hair, others from another country may be more concerned with their waist or height, as suggested by the items with the greatest non-invariance of the BASS. In line with this suggestion, Mellor et al. (2013) and Mellor et al. (2014) have shown that different body parts are associated with body dissatisfaction among adolescent females and males from Australia, China and Malaysia. Past research has also suggested cross-cultural differences in attitudes toward body fat, obesity, and thinness (Swami, 2015). 


\subsection{Impact of gender, BMI and perceived SES on response patterns}

For the emerging adults participating in this study, gender and BMI negatively predicted scores on the BAS-2 and the BASS, and positively predicted those on the PACS and the WBIS-M. That is, women and participants with higher BMIs tended to score lower on body satisfaction and appreciation. Additionally, they tended to rate themselves with higher levels of weight bias internalization and to report more physical appearance comparison. With regards to gender, our results are not surprising considering other studies have also found that young adult women are more likely than men to be preoccupied and dissatisfied with their appearance (Heiman \& Olenik-Shemesh, 2019). However, research comparing the facets of positive body image in women and men is still rare and has led to mixed results (Tiggemann, 2015). While some authors concluded that men and women experience and conceptualize body appreciation in a similar way (Tylka \& Wood-Barcalow, 2015a), others assert that it is higher in men than in women of various countries (Tiggemann, 2015). Our results support the idea that men of various cultural backgrounds and living in different countries may have more flexible body ideals and be more inclined to focus on their bodies' functionality and abilities (Tiggemann, 2015). However, cross-country consideration of other facets of gender orientation and sexuality may expand upon these findings. The inverse association between higher BMI and body appreciation found in our study has also been documented elsewhere, across different cultural and ethnic groups (Tylka \& Wood-Barcalow, 2015a). Previous research suggests this association is nurtured by internalized weight bias (Tylka \& Wood-Barcalow, 2015a). It seems likely that prevailing anti-fat attitudes in emerging adults as well as a tendency to compare their appearance to the one of others act in a reciprocal way with positive body image in individuals with higher BMI (Homan \& Tylka, 2015).

Perceived SES was associated with higher BAS-2 and BASS scores and lower PACS and WBIS-M scores, indicating that participants who perceived themselves as having higher SES reported higher body appreciation, as well as less appearance comparison and weight bias. A more objective measure of SES could lead to slightly different results and would be interesting to consider in future research. That being said, perceived and objective SES could be reflective of higher education levels, which have also been linked to higher BAS scores (Swami, Hadji-Michael, \& Furnham, 2008). According to Swami et al. (2008), higher education and greater financial means can help individuals to attain a more positive body image through better access to psychological and physical health resources. Taken together, our MIMIC findings indicate that it is important to include gender, BMI, and perceived SES as covariates and to control for these demographics when comparing scores on the BAS-2, BASS, PACS, and WBIS-M across countries.

\subsection{Strengths and limitations}

This study examined measures associated with positive body image across a large sample of women and men derived from eight different countries. It contributes to the study of positive body image by addressing the lack of cross-validation information and by providing measurement information about four related constructs: body appreciation, body appearance satisfaction, physical appearance comparisons, and weight bias internalization. Results indicate that the BAS-2, BASS, PACS, and WBIS-M can be used for comparison among different samples of English and non-English speaking participants. To our knowledge, no other study has considered multiple facets of positive body image, physical appearance comparisons and weight bias among such a large sample of emerging adults.
However, some limitations are worth noting. First, it is unlikely that each subsample from a different country is representative of all individuals residing in that country. Some intra-country differences could be possible. Second, our participants were purposefully chosen to be emerging adults, an age period in which adults display less body appreciation and are less oriented towards their body's functionality (Augustus-Horvath \& Tylka, 2011). Moreover, pregnant women, who may be more appreciative of their body and its functionality, were excluded (Watson, Fuller-Tyszkiewicz, Broadbent, \& Skouteris, 2015). Third, participants were mostly students. Despite the various recruitment means we used, communities of emerging adults were difficult to reach and incentives for participation such as course credits were not applicable for nonstudents. Fourth, although this study offers evidence of measurement invariance, this conclusion was reached after multiple iterations of analyses, which indicates, as mentioned earlier, some differences across countries that should be further studied. Future research could expand on our work by including a broader age range of participants, and an even larger, more representative sample of individuals from different countries. Further, cross-cultural qualitative research may deepen our understanding of individuals' perceptions of the items and surveys used in this research.

\subsection{Conclusions}

Positive body image is a relatively new concept, distinct from body dissatisfaction (Tylka \& Wood-Barcalow, 2015a). It includes multiple facets, such as body satisfaction and appreciation, and implies some reciprocity between an individual and the environment, which can take the form of weight bias internalization and physical appearance comparison. Cross-country assessment, in English and non-English samples, is necessary to determine whether measures related to positive body image like the BAS-2, BASS, and other measures like the PACS, and WBIS-M might be efficient and accessible tools to use for research and clinical purposes. This study indicates partial measurement invariance of these four questionnaires across Australia, Belgium, Canada, China, Italy, Japan, Spain, and the United States. Even though some non-invariance was found, it appears these measures are sensitive to contextual variations and can be used for comparative research. Further cross-cultural research is needed and the understanding of the potential causes of the partial invariance identified in this study should be pursued. Overall, our findings should help to pave the way for future research examining positive body image among individuals from diverse countries. As Tylka and Wood-Barcalow (2015a) noted, although the study of positive body image is flourishing, there is a great deal that remains unknown about the causes, consequences, and correlates of it. With the scales demonstrating strong psychometric properties across different cultural groups, the cross-cultural study of these important areas can move forward.

\section{Funding}

This research did not receive any specific grant from funding agencies in the public, commercial, or not-for-profit sectors.

\section{Uncited references}

Halliwell (2013) and Lai and Green (2016).

\section{Declaration of Competing Interest}

The authors report no declarations of interest.

\section{Acknowledgements}

The authors would like to acknowledge the central role that Lina Ricciardelli played in the conceptualization of this study and to the 
broader field of body image. The last author wants to thank Alexandre J.S. Morin for his statistical advice.

\section{Appendix A. Supplementary data}

Supplementary material related to this article can be found, in the online version, at doi:https://doi.org/10.1016/j.bodyim.2020.09.014.

\section{References}

Aimé, A., Villatte, A., Cyr, C., \& Marcotte, D. (2017). Can weight predict academic performance in college students? An analysis of college women's self-efficacy, absenteeism, and depressive symptoms as mediators. Journal of American College Health, 65, 168-176. doi:10.1080/07448481.2016.1266639.

Andrew, R., Tiggemann, M., \& Clark, L. (2016). Predicting body appreciation in young women: An integrated model of positive body image. Body Image, 18, 34-42. doi:10.1016/j.bodyim.2016.04.003.

Arnett, J.J., Žukauskienè, R., \& Sugimura, K. (2014). The new life stage of emerging adulthood at ages 18-29 years: Implications for mental health. The Lancet Psychiatry, 1(7), 569-576. doi:10.1016/S2215-0366(14)00080-7.

Augustus-Horvath, C.L., \& Tylka, T.L. (2011). The acceptance model of intuitive eating: A comparison of women in emerging adulthood, early adulthood, and middle adulthood. Journal of Counseling Psychology, 58, 110-125. doi:10.1037/a0022129.

Avalos, L.C., Tylka, T.L., \& Wood-Barcalow, N. (2005). The Body Appreciation Scale: Development and psychometric evaluation. Body Image, 2, 285-297. doi:10.1016/ j.bodyim.2005.06.002.

Brown, T.A., Cash, T.F., \& Mikulka, P.J. (1990). Attitudinal body-image assessment: Factor analysis of the body-self relations questionnaire. Journal of Personality Assessment, 55(1-2), 135-144. doi:10.1080/00223891.1990.9674053.

Cash, T.F. (2000). The multidimensional body-self relations questionnaire Available from the author at (3rd ed.). www.body-images.com

Chen, F.F. (2007). Sensitivity of goodness of fit indexes to lack of measurement. Structural Equation Modeling, 14, 464-504. doi:10.1080/10705510701301834.

Cheung, G.W., \& Rensvold, R.B. (2002). Evaluating goodness-of fit indexes for testing measurement invariance. Structural Equation Modeling, 9, 233-255. doi:10.1207/ S15328007SEM0902_5.

Durso, L.E., \& Latner, J.D. (2008). Understanding self-directed stigma: Development of the weight bias internalization scale. Obesity, 16(S2), S80-S86. doi:10.1038/ oby.2008.448.

Fallon, E.A., Harris, B.S., \& Johnson, P. (2014). Prevalence of body dissatisfaction among a United States adult sample. Eating Behaviors, 15(1), 151-158. doi:10.1016/ j.eatbeh.2013.11.007.

Fiske, A.P. (2020). The lexical fallacy in emotion research: Mistaking vernacular words for psychological entities. Psychological Review, 127(1), 95-113. doi:10.1037/ rev0000174.

Fiske, L., Fallon, E.A., Blissmer, B., \& Redding, C.A. (2014). Prevalence of body dissatisfaction among United States adults: Review and recommendations for future research. Eating Behaviors, 15, 357-365. doi:10.1016/j.eatbeh.2014.04.010.

Flegal, K.M., Kruszon-Moran, D., Carroll, M.D., Fryar, C.D., \& Ogden, C.L. (2016). Trends in obesity among adults in the United States, 2005 to 2014. JAMA, 315(21), 2284-2291. doi:10.1001/jama.2016.6458.

Fuller-Tyszkiewicz, M., Chhouk, J., McCann, L.A., Urbina, G., Vuo, H., Krug, I., .. Richardson, B. (2019). Appearance comparison and other appearance-related influences on body dissatisfaction in everyday life. Body Image, 28, 101-109. doi:10.1016/j.bodyim.2019.01.002.

Grabe, S., \& Hyde, J.S. (2006). Ethnicity and body dissatisfaction among women in the United States: A meta-analysis. Psychological Bulletin, 132(4), 622-640. doi:10.1037/ 0033-2909.132.4.622.

Halliwell, E. (2013). The impact of thin idealized media images on body satisfaction: Does body appreciation protect women from negative effects? Body Image, 10, 509-514. doi:10.1016/j.bodyim.2013.07.004.

Halliwell, E. (2015). Future directions for positive body image research. Body Image, 14, 177-189. doi:10.1016/j.bodyim.2015.03.003.

Heiman, T., \& Olenik-Shemesh, D. (2019). Perceived body appearance and eating habits: The voice of young and adult students attending higher education. International Journal of Environmental Research and Public Health, 16(3), 451. doi:10.3390/ ijerph16030451.

Homan, K.J., \& Tylka, T.L. (2015). Self-compassion moderates body comparison and appearance self-worth's inverse relationships with body appreciation. Body Image, 15, 1-7. doi:10.1016/j.bodyim.2015.04.007.

Hu, L., \& Bentler, P.M. (1999). Cutoff criteria for fit indexes in covariance structure analysis: Conventional criteria vs new alternatives. Structural Equation Modeling, 6, 1-55. doi:10.1080/10705519909540118.

Kelly, M.C., \& Latner, J.D. (2015). Evaluating patterns of weight and body composition change among college women. Eating Behaviors, 17, 157-162. doi:10.1016/ j.eatbeh.2015.01.016.

Kelly, N.R., Mitchell, K.S., Gow, R.W., Trace, S.E., Lydecker, J.A., Bair, C.E., \& Mazzeo, S. (2012). An evaluation of the reliability and construct validity of eating disorder measures in white and black women. Psychological Assessment, 24(3), 608-617. doi:10.1037/a0026457.

Lai, K., \& Green, S.B. (2016). The problem with having two watches: Assessment of fit when RMSEA and CFI disagree. Multivariate Behavioral Research, 51(2), 1-20. doi:10.1080/00273171.2015.1134306.
Marsh, H.W., Hau, K.-T., \& Grayson, D. (2005). Goodness of fit evaluation in structural equation modeling. In Maydeu-Olivares, A., \& McArdle, J. (Eds.), Contemporary psychometrics. A Festschrift for Roderick P. McDonald. Mahwah, NJ: Erlbaum.

Marsh, H.W., Nagengast, B., \& Morin, A.J.S. (2013). Measurement invariance of big-five factors over the life span: ESEM tests of gender, age, plasticity, maturity, and la dolce vita effects. Developmental Psychology, 49, 1194-1218. doi:10.1037/a0026913.

McCabe, M., Tatangelo, G., Watson, B., Fuller-Tyszkiewicz, M., Rodgers, R.F., Aimé, A., .. Ricciardelli, L. (2019). Development and testing of a model for risk and protective factors for eating disorders and higher weight among emerging adults: A study protocol. Body Image, 31, 139-149. doi:10.1016/j.bodyim.2019.10.001.

McDonald, R.P. (1970). Theoretical foundations of principal factor analysis, canonical factor analysis, and alpha factor analysis. British Journal of Mathematical \& Statistical Psychology, 23, 1-21. doi:10.1111/j.2044-8317.1970.tb00432.x.

Mellor, D., Waterhouse, M., Hidayah, N., Cochrane, J., Xu, X., McCabe, M., ... Ricciardelli, L. (2013). Which body features are associated with female adolescents' body dissatisfaction? A cross-cultural study in Australia, China and Malaysia. Body Image, 10, 54-61. doi:10.1016/j.bodyim.2012.10.002.

Mellor, D., Hucker, A., Waterhouse, M., Hidayah bt Mamat, N., Xu, X., Cochrane, J., ... Ricciardelli, L (2014). A cross-cultural study investigating body features associated with male adolescents' body dissatisfaction in Australia, China and Malaysia. American Journal of Men's Health, 8, 521-531. https://doi.org/ $10.1177 \% 2$ F1557988314528370.

Meredith, W. (1993). Measurement invariance, factor analysis and factorial invariance. Psychometrika, 58, 525-543. doi:10.1007/BF02294825.

Morin, A.J.S., Marsh, H.W., \& Nagengast, B. (2013). Exploratory structural equation modeling. In Hancock, G.R., \& Mueller, R.O. (Eds.), Structural equation modeling: A second course (2nd ed., pp. 395-438). Charlotte, NC: Information Age.

Morin, A.J., Maïano, C., Scalas, L.F., Aşçı, F.H., Boughattas, W., Abid, S., ... Probst, M. (2018). Cross-cultural validation of the short form of the Physical Self Inventory (PSI-S). Sport, Exercise, and Performance Psychology, 7(1), 60-79. doi:10.1037/ spy0000096.

Muthén, L.K., \& Muthén, B. (2018). Mplus user's guide (8th ed.). Los Angeles, CA: Muthén \& Muthén.

Ng, S.-K., Barron, D., \& Swami, V. (2015). Factor structure and psychometric properties of the Body Appreciation Scale among adults in Hong Kong. Body Image, 13, 1-8. doi:10.1016/j.bodyim.2014.10.009.

Operario, D., Adler, N.E., \& Williams, D.R. (2004). Subjective social status: Reliability and predictive utility for global health. Psychology \& Health, 19(2), 237-246. doi:10.1080/08870440310001638098.

Pearl, R.L., \& Puhl, R.M. (2014). Measuring internalized weight attitudes across body weight categories: Validation of the modified Weight Bias Internalization Scale. Body Image, 11(1), 89-92. doi:10.1016/j.bodyim.2013.09.005.

Piran, N. (2015). New possibilities in the prevention of eating disorders: The introduction of positive body image measures. Body Image, 14, 146-157. doi:10.1016/ j.bodyim.2015.03.008.

Rodgers, R.F., Franko, D.L., Donovan, E., Cousineau, T., Yates, K., McGowan, K., ... Lowy, A.S. (2017). Body image in emerging adults: The protective role of self-compassion. Body Image, 22, 148-155. doi:10.1016/j.bodyim.2017.07.003.

Singer, E., \& Bossarte, R.M. (2006). Incentives for survey participation: When are they "coercive"? American Journal of Preventive Medicine, 31(5), 411-418. doi:10.1016/ j.amepre.2006.07.013.

Swami, V. (2015). Cultural influences on body size ideals: Unpacking the impact of westernization and modernization. European Psychologist, 20(1), 44-51. doi:10.1027/1016-9040/a000150.

Swami, V., Hadji-Michael, M., \& Furnham, A. (2008). Personality and individual difference correlates of positive body image. Body Image, 5(3), 322-325. doi:10.1016/ j.bodyim.2008.03.007.

Swami, V., Airs, N., Chouhan, B., Leon, M.A.P., \& Towell, T. (2009). Are there ethnic differences in positive body image among female British undergraduates? European Psychologist, 14, 288-296. doi:10.1027/1016-9040.14.4.288.

Swami, V., Frederick, D.A., Aavik, T., Alcalay, L., Allik, J., Anderson, D., .. Zivcic-Becirevic, I. (2010). Body weight ideals and body dissatisfaction in 26 countries across 10 world regions: Results of the International Body Project I. Personality and Social Psychology Bulletin, 36, 309-325. https://doi.org/ $10.1177 \% 2 F 0146167209359702$.

Swami, V., Khatib, N.A.M., Toh, E., Zahari, H.S., Todd, J., \& Barron, D. (2019). Factor structure and psychometric properties of a Bahasa Malaysia (Malay) translation of the Body Appreciation Scale-2 (BAS-2). Body Image, 28, 66-75. doi:10.1016/ j.bodyim.2018.12.006.

Swanson, J.A. (2016). Trends in literature about emerging adulthood: Review of empirical studies. Emerging Adulthood, 4(6), 391-402. doi:10.1177/2167696816630468.

Thompson, J.K., Heinberg, L., \& Tantleff-Dunn, S. (1991). The physical appearance comparison scale. The Behavior Therapist, 14, 174. https://scholarcommons.usf.edu/ psy_facpub/2116.

Tiggemann, M. (2015). Considerations of positive body image across various social identities and special populations. Body Image, 14, 168-176. doi:10.1016/ j.bodyim.2015.03.002.

Tylka, T.L., \& Wood-Barcalow, N.L. (2015). What is and what I not positive body image? Conceptual foundations and construct definition. Body Image, 14, 118-129. doi:10.1016/j.bodyim.2015.04.001

Tylka, T.L., \& Wood-Barcalow, N.L. (2015). The Body Appreciation Scale-2: Item refinement and psychometric evaluation. Body Image, 12, 53-67. doi:10.1016/ j.bodyim.2014.09.006. 
Untas, A., Koleck, M., Rascle, N., \& Borteyrou, X. (2009). Psychometric properties of the French adaptation of the Multidimensional Body Self-Relations Questionnaires Appearance Scales. Psychological Reports, 105, 461-471. doi:10.2466/ PR0.105.2.461-471.

Wasylkiw, L., \& Butler, N.A. (2014). Body talk among undergraduate women: Why conversations about exercise and weight loss differentially predict body appreciation. Journal of Health Psychology, 19(8), 1013-1024. doi:10.1177/1359105313483155.

Watson, B., Fuller-Tyszkiewicz, M., Broadbent, J., \& Skouteris, H. (2015). The meaning of body image experiences during the perinatal period: A systematic review of the qualitative literature. Body Image, 14, 102-113. doi:10.1016/j.bodyim.2015.04.005.

Webb, J.B., Wood-Barcalow, N.L., \& Tylka, T.L. (2015). Assessing positive body image: Contemporary approaches and future directions. Body Image, 14, 130-145. doi:10.1016/j.bodyim.2015.03.010.

Wood-Bacalow, N.L., Tylka, T.L., \& Augustus-Horvarth, C.L. (2010). But I like my body": Positive body image characteristics and a holistic model for young-adult women. Body Image, 7, 106-116. doi:10.1016/j.bodyim.2010.01.001 